\title{
Chitosan-based inks: 3D printing and bioprinting strategies to improve shape fidelity, mechanical properties, and biocompatibility of 3D scaffolds \\ Premio Congreso SIBB 2018
}

\author{
A. Mora-Boza ${ }^{1}$, M.K. Włodarczyk-Biegun ${ }^{2}$, A. del Campo ${ }^{2,3}$, B. Vázquez-Lasa ${ }^{1}$, J. San Román ${ }^{1}$ \\ ${ }^{1}$ Institute of Polymer Science and Technology, ICTP-CSIC, and CIBER BNN, Madrid, Spain \\ ${ }^{2}$ Leibniz-Institute for New Materials, Saarbrücken, Germany \\ ${ }^{3}$ Chemistry Department, Saarland University, Saarbrücken, Germany
}

\begin{abstract}
Resumen
El campo de la impresión y la bioimpresión $3 D$ ha alcanzado un gran desarrollo en los últimos años. La llegada de estas técnicas de biofabricación a la ingeniería tisular han supuesto una revolución debido a las estructuras biomiméticas tan complejas que permiten sintetizar. El uso del quitosano como componente de tintas basadas en polímeros naturales es muy atractivo debido a las propiedades beneficiosas que presenta (alta biocompatibilidad, biodegradabilidad, bajo coste). Sin embargo, su aplicación se ve limitada por su baja solubilidad en condiciones fisiológicas y sus pobres propiedades mecánicas. En este artículo, revisamos el estado del arte relacionado con las estrategias actuales de impresión $3 D$ que hacen uso de tintas y biotintas basadas en quitosano para el desarrollo de soportes biomiméticos. También analizamos las estrategias de entrecruzamiento que se aplican actualmente para mejorar su printabilidad, resaltando además los derivados de quitosano disponibles que permiten encapsular células para bioimpresión 3D, incluyendo además nuestra contribución al campo. Prevemos que el uso de quitosano en la impresión 3D aumentará significativamente en los próximos años gracias a los esfuerzos que se están desarrollando en términos de mecanismos de gelificación y derivados de quitosano que permiten la encapsulación celular.
\end{abstract}

Palabras clave: quitosano, impresión 3D, fidelidad de forma, ingeniería tisular, procesos de gelificación

\begin{abstract}
$3 D$ printing and bioprinting fields have experienced a rapid development in the last years. The arrival of this biofabrication technology to tissue engineering applications has revolutionized the field, since highly complex and biomimetic constructs can be synthetized. Chitosan is a promising naturalderived polysaccharide to be used as ink because of their attractive properties (e.g. biodegradability, biocompatibility, low cost). Nevertheless, its application has been limited due to their poor solubility properties at physiological conditions and soft stiffness, which significantly reduces shape fidelity and resolution of the printed scaffolds. This paper reviews the state-of-the-art in terms of $3 D$ printing technology using chitosan-based inks and bio-inks. Printing and crosslinking strategies applied to improve printability of the inks are also reviewed, highlighting the current available chitosan derivatives that can be used for cell-encapsulation in 3D bioprinting. Finally, we also describe our contribution to the field using a novel crosslinker compound to improve shape fidelity and biological features of $3 D$ printed scaffolds. We envision that the use of chitosan in $3 D$ printing will significantly increase over the next years thanks to the many efforts are being made in terms of development of new gelation mechanisms and chitosan derivatives that allow cell encapsulation.
\end{abstract}

Keywords: chitosan, 3D printing, shape fidelity, tissue engineering, gelation process

Correspondencia: Ana Mora-Boza. ana.mora@ictp.csic.es 


\section{Acronyms List}

$3 \mathrm{D}$ - three-dimensional

ECM - extracellular matrix

TPP - tripolyphosphate

HBC - hydroxybutyl-chitosan

GelMA - methacrylated gelatin

UV - ultraviolet

CMC - Carboxymethyl-chitosan

MSCs - mesenchymal stem cells

iPSCs - induced pluripotent stem cells

$\beta$-GP - $\beta$-glycerolphosphate

\section{Introduction}

Three-dimensional (3D) printing has arisen in the last years as a promising tool for the development of biofunctional and biomimetic 3D scaffolds for tissue engineering applications. 3D printing is an additive manufacturing technique that allows the fabrication of extremely precise structures with high degree of complexity and meticulous details at the micrometer level $[1,2]$. Moreover, cell-laden scaffolds can be also synthetized by using bioinks, which are the combination of printable materials and living cells. This process is termed 3D bioprinting or cellular 3D printing $[1,3,4]$.

3D printing provides important advantages that can play a pivotal role in the effective regeneration of tissues respect to traditional fabrication techniques (e.g. solvent casting, freeze drying, electrospinning). These conventional methods usually lead to uncontrolled and random pore distribution since they are not able to fabricate accurate pore sizes and geometries, which also result in low interconnectivity [2]. Moreover, inaccurate cell distribution due to random geometries generated by traditional methodologies can be problematic because cell growing and differentiation processes usually need from precise arrangements depending on the tissue function [2, 4]. For example, endothelial cells need from aligned structures for the development of blood vessels or osteoblasts arrange to form mineralized clusters [2] or other tissues, like cartilage, are characterized by hierarchical structures with very specialized cells in each layer $[4,5]$.
$3 \mathrm{D}$ printing has traced a continuous evolution in the last decades to overcome these limitations and brings the opportunity to fabricate scaffolds that promote functional tissue regeneration. Nevertheless, researching on novel biocompatible inks and printing strategies that fulfil the main biofabrication requirements is still necessary for the progress of the field.

Hydrogels have been traditionally used for tissue engineering applications due to their excellent biocompatibility, since they recapitulate the natural extracellular matrix (ECM) and provide a highly hydrated environment for mechanical and biological cell support [4]. Many efforts have been made to enhance bioactivity and biocompatibility of hydrogels for functional tissue regeneration, but little attention has been paid to the improvement of their physico-chemical and mechanical properties $[1,3,4]$. The bottleneck of hydrogelbased inks use in 3D printing is their lack of printability due to their high-water content which leads to poor mechanical properties and processability. Printability is a key factor regarding $3 \mathrm{D}$ printing because it is an essential property to achieve high resolution and shape fidelity. Thus, fast crosslinking mechanisms are interesting to improve printability of hydrogels because a fast phase transition (from a liquid-like solution to a solid state) will improve manufacturing process $[3,6]$. Finally, a suitable ink must also exhibit shear-thinning properties while printing, adequate mechanical stability for maintaining the shape after printing, and good structural integrity at physiological conditions. Moreover, they should be cytocompatible if cells are encapsulated and printed along with the hydrogel [7].

Chitosan is a natural polysaccharide obtained from the alkaline deacetylation of chitin, and composed of randomly distributed $\beta$ - $(1 \rightarrow 4)$-linked D-glucosamine (deacetylated unit) and $\mathrm{N}$-acetyl-D-glucosamine (acetylated unit) (Figure 1) $[6,8]$. Chitosan is biodegradable, biocompatible, non-toxic, and its origin makes it a renewable and eco-friendly material [8]. Chitosan exhibits a pKa value of 6.2 , which means that below this $\mathrm{pH}$, amino groups $\left(\mathrm{NH}_{2}\right)$ of chitosan chains are protona- 
ted $\left(\mathrm{NH}_{3}{ }^{+}\right)$, and the polymer becomes cationic and soluble. Above this $\mathrm{pH}$ chitosan remains insoluble (Figure 1). In addition, positive charges of chitosan allow it to interact with a wide range of molecules. However, chitosan is characterised by weak mechanical properties, specially under hydrated conditions [8]. Thus, numerous efforts have been made to improve the mechanical strength of chitosan by the incorporation of additives [9-14] or the application of different gelation methods [15]. Specifically, chitosan can experience a sol-gel transition by different gelation processes which can be ionotropic, mediated by crosslinkers, or complexation with other polymers (polyelectrolyte formation) [15].

Although the number of studies that use chitosan in the 3D printing field has increased exponentially in the latest ten years, the application of this polymer to this technology remains relatively low [16]. Some last studies have claimed the use of chitosan instead of alginate (i.e. the quintessential hydrogel-based printable material) due to its better properties regarding cellular proliferation and differentiation [17]. Here, we describe the most recently applied printing techniques and crosslinking methods for chitosan in $3 \mathrm{D}$ (bio)printing field. We also highlight the implemented chemical modifications to enhance the use of chitosan in bioprinting applications, including our contribution to the field using chitosan-based inks. We envision that the further implementation of chitosan to printing technology will open a new door to the used of hydrogel-based inks for the fabrication of complex structures with promising applications in tissue engineering and drug delivery applications.

\section{D printing approaches for the use of chito- san-based inks}

Hydrogel-based inks printability remains a challenge due to their stiffness and shrink tendency, which is one of the limiting factors in terms of shape fidelity. In this context, several strategies have been explored to improve hydrogel printing accuracy in the last years. One approach is the use of high viscous polymeric solutions, which are usually useful to obtain solid filaments that allow the deposition of robust hydrogel scaffolds with self-supporting capacity $[4,18]$. Viscous inks can be obtained by: (i) increasing total polymer concentration, (ii) using polymers with high molecular

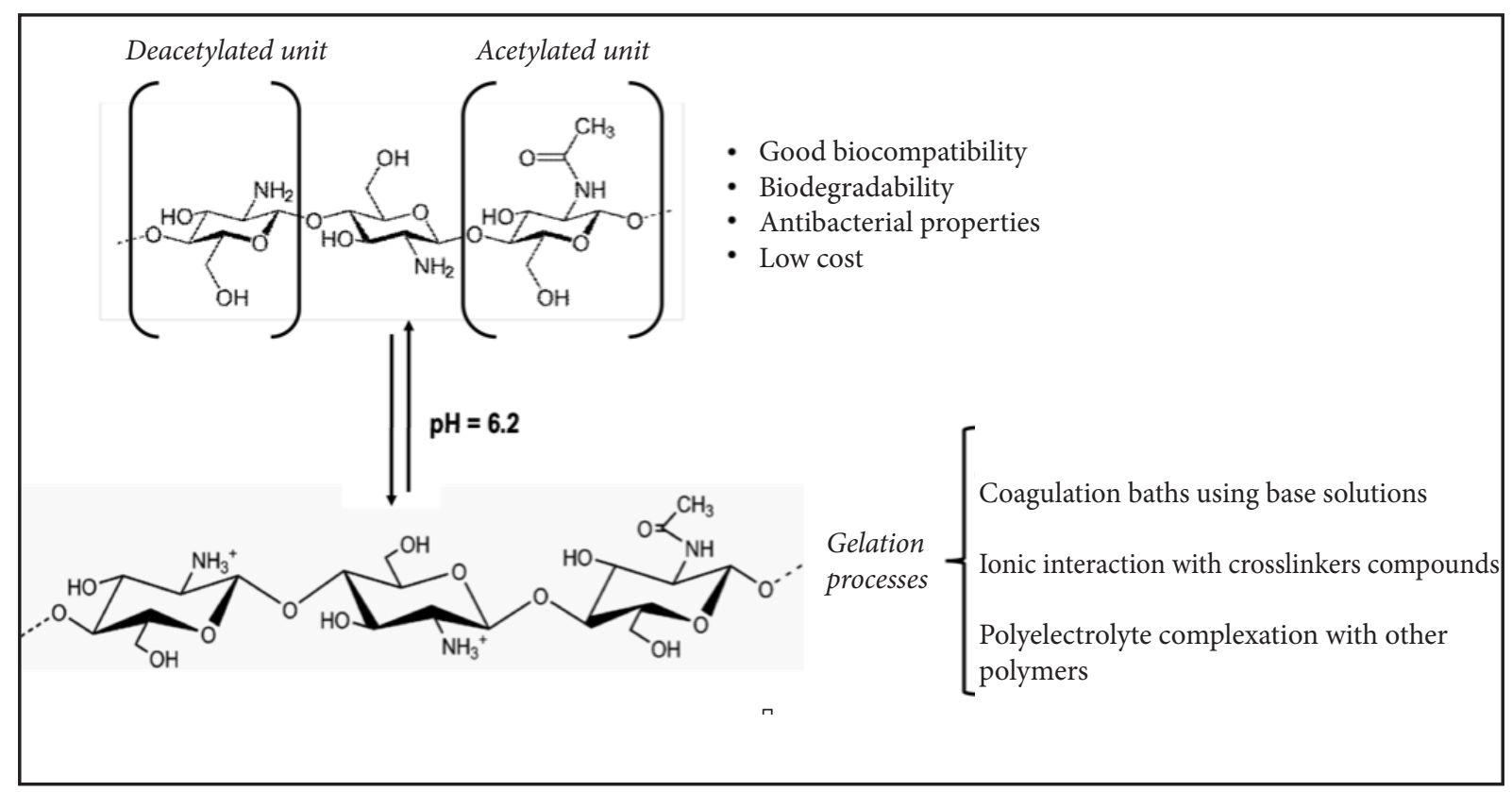

Figure 1. Chitosan chemical structure and transition phase at pH 6.2. 
weight, or (ii) combining two different polymers that can form polyelectrolyte complexes [4]. Other approaches to enhance 3D printing deposition with high resolution are more focused on the development and optimization of crosslinking techniques which allow a fast sol-gel transition [15]. This approach is in somewhat preferably in comparison to high viscous solutions, since a low polymer content $(<10 \mathrm{wt}-\%)$ will provide a lesser dense polymeric network with improved cellular performance [4].

The characteristic $\mathrm{pH}$-sensitivity of chitosan provides an excellent opportunity to explore its gelation abilities, which have been successfully adapted to 3D printing methodologies. Moreover, crosslinking and neutralization of chitosan to neutral $\mathrm{pH}$ is necessary previously to cell seeding [19].

\section{Neutralization baths}

As it has been explained before, chitosan phase rapidly precipitates at $\mathrm{pH}$ above 6.2. This transition from soluble to insoluble results in the formation of a hydrogel due to the neutralization of amino groups present in chitosan chains. Thus, hydrogen bonding and hydrophobic chain interactions are formed. However, neutralization steps are usually accompanied by shrinkage processes which significantly reduce the shape fidelity and resolution of the printed scaffolds $[6,18]$. To avoid this effect, Zhang et al. [18] proposed the incorporation of silk particles to chitosan solution to enhance mechanical properties and reduce post-shrinkage due to coagulation in 12 wt- $\% \mathrm{NaOH}$ solution after printing each layer. $3 \mathrm{D}$ printed scaffolds showed improved postprinting fidelity and mechanical properties, since compressive modulus increased up to 5 -fold after silk particles reinforcement. Wu et al. [6] analysed in their study the effect of neutralization steps on the mechanical properties and printing fidelity of very intricate structures. They used very concentrated chitosan solution (10 wt-\%) that was dissolved in an acidic mixture, and 3D printed scaffolds were immersed in a $1 \mathrm{M} \mathrm{NaOH}$ solution as coagulation bath. In this work, the authors were able to fabricate complex scaffolds with very high resolution $(\approx 30 \mu \mathrm{m})$. In other interesting work, Almeida et al. [20] analysed the cytokine secretion profile of seeded macrophages in chitosan 3D printed scaffolds that were neutralised after immersion in a $8 \mathrm{wt}-\% \mathrm{NaOH}$ solution. They surprisingly found out that macrophage responses were dependent on specific parameters of the $3 \mathrm{D}$ printed structures (e.g. chemistry, topography, architecture).

Freeze deposition methodology combined with subsequent neutralization steps have been also widely applied for the 3D printing of chitosan-based inks [15, 21, 22]. Elviri et al. [21] prepared chitosan scaffolds by their deposition in a cryogenic chamber followed by gelation in a $8 \mathrm{wt}-\% \mathrm{KOH}$ solution. In addition, they used raffinose as viscosity modifier. Fibroblasts were seeded on the top of the 3D printed scaffolds and showed excellent cell adhesion and proliferation. This approach was further optimized and applied in diabetic rats for wound healing evaluation by Intini et al. [22]. Wounds treated with their 3D chitosanraffinose printed scaffolds demonstrated excellent tissue regeneration and functionality respect to commercially available products.

On the contrary, although shrinkage after $3 \mathrm{D}$ printing is not desirable to maintain high shape fidelity, it is directly related to hydration capacity, which is an essential property to be evaluated for tissue engineering applications. Since proteins and bioactive compounds adsorption will depend on swelling ratio, this property is crucial to mimic the native ECM and enhance biocompatibility of the 3D printed scaffolds. Bergonzi et al. [15] carried out a profound study where 3D printed scaffolds were neutralized with three different gelation solutions: $\mathrm{KOH}, \mathrm{Na}_{2} \mathrm{CO}_{3}$, and ammonia vapours. The authors concluded that physical properties and stability at physiological conditions depended deeply on applied coagulation media, and therefore they should be chosen in function of the target area of the body to be repaired.

\section{Ionotropic and crosslinker-mediated gelation}

Cationic nature of chitosan provides enough positive charges under acidic conditions to electrostatically interact with negatively char- 
ged ions, leading to the formation of a physically crosslinked polymeric network. There is a huge variety of compounds and molecules that can be applied for chitosan crosslinking or gelation (e.g. citrates, phosphates such as tripolyphosphate (TPP). The type of molecule will have an influence on the swelling and mechanical properties of the formed hydrogel [15]. Nevertheless, the crosslinking kinetics should be fast for its successful application in 3D printing technology to ensure resolution and high printing fidelity [4].

Wang et al. [5] fabricated multi-layered (i.e. consecutive layers) hydrogels scaffolds using hydroxybutyl-chitosan (HBC), a thermosensitive derivative of chitosan, and a one-step ionic post-treatment without the necessity of using strong base solutions. They evaluated the use of different salts and concentrations, concluding that $3 \mathrm{D}$ printed scaffolds treated with a 10 wt- $\% \mathrm{NaCl}$ solution showed the best structural, mechanical and biological features. In other interesting work, Hafezi et al. [23] synthetized 3D printed chitosan scaffolds crosslinked with genipin for wound chronic healing applications. Genipin is an ionic biocompatible crosslinker with low cytotoxicity, anti-inflammatory and antibacterial properties [24].

In our group, we synthetized a novel bioactive ionic crosslinker name glycerylphytate [25] that was successfully applied in 3D printing technology [7]. In this work, multi-layered $3 \mathrm{D}$ printed scaffolds were fabricated using a dual-step crosslinking process. A blend ink of low polymer content ( $8 \mathrm{wt}-\%$ ) composed of methacrylated gelatin (GelMA) and chitosan was first irradiated with ultraviolet (UV) light to carry out photopolymerization of GelMA, followed by ionic crosslinking of amino groups present in chitosan and GelMA with glycerylphytate without the necessity of harsh neutralization or several washing steps. Thus, 3D scaffolds with excellent shape fidelity and long-term stability were obtained (Figure 2a). Moreover, these scaffolds showed improved biological features in comparison to $3 \mathrm{D}$ printed scaffolds that were crosslinked with the traditionally used TPP (Figure $2 \mathrm{~b}$ ), which is also a phosphate-based ionic crosslinker. Specifically, glycerylphytate-crossslinked scaffolds showed higher L929 fibroblasts adhesion and proliferation over time than TPP-crosslinked scaffolds [7].

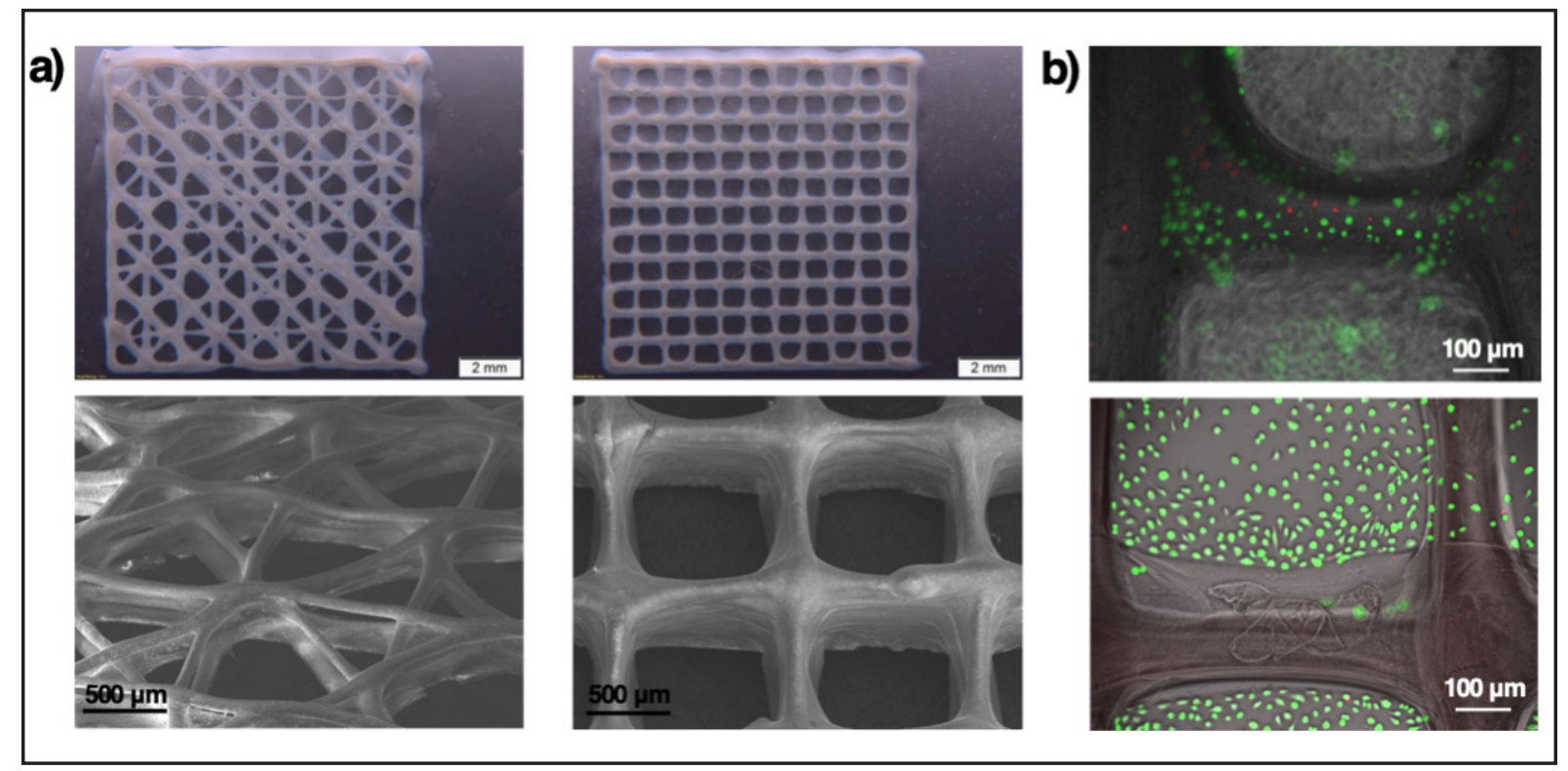

Figure 2. a) Light microscopy pictures (top) and scanning electron micrographs (bottom) of multilayered 3D printed scaffolds with different geometries using dual-step crosslinking processes consisted of UV light irradiation and immersion in glycerylphytate solution for ionic crosslinking; b) Fluorescence pictures of live/dead assay performed on glycerylphytate-crosslinked scaffold (top) and TPP-crosslinked scaffold (bottom) after 24 hours of L929 fibroblasts seeding. 


\section{Polyelectrolyte complexes formation}

The formation of chitosan polyelectrolyte complexes is an interesting approach to improve mechanical properties of hydrogelbased inks by the blending of different polymers (e.g. gelatin, alginate) that can provide additional features to the systems than when they are used individually [15]. The formation of polyelectrolyte complexes involves electrostatic interactions between the mixed polymers, which exhibit opposite charges. Liu et al. [26] obtained 3D printed scaffolds from an alginate-chitosan polyion complexes ink. The addition of chitosan to the polyanionic alginate was useful to increase the viscosity of alginate solution. Thus, 3D structures with excellent shape fidelity and improved mechanical strength were obtained. $\mathrm{Ng}$ et al. $[27,28]$ used gelatin-chitosan polyelectrolyte hydrogels for the $3 \mathrm{D}$ printing of skin constructs. The blend ink also exhibited higher viscosity due to the combination of both polymers that eased the 3D deposition of complex structures. In our work, where a GelMA/chitosan ink was crosslinked with UV light and glycerylphytate crosslinker, we observed a similar behaviour. Viscosity of the blend between GelMA and chitosan increased the viscosity of the ink solution respect to those of individual components, obtaining a self-standing filament that eased 3D deposition but still having a low concentrated ink ( $8 \mathrm{wt}-\%$ of total polymer content) [7]. This is an interesting approach not only because different polymers can be combined and provided their respective benefits to the whole system, but also because high viscosities can be achieved with relatively low polymer content attributed to interactions of polyelectrolytic type. This strategy will finally enhance printability of blend hydrogel solutions.

\section{Photocrosslinkable chitosan derivatives}

Photopolymerization is an interesting technique for the development of stable covalent-crosslinked hydrogels. In the last years, UV-crosslinkable chitosan derivatives have been developed and successfully applied in $3 \mathrm{D}$ printing $[1,11,29,30]$. Sayyar et al.
[11] carried out an interesting work where they combined photocrosslinkable chitosan and chemically converted graphene for the synthesis of 3D printed conducting scaffolds. The addition of graphene to the composites improved not only mechanical properties of chitosan scaffolds but also their biocompatibility, as L929 murine fibroblasts showed higher cell adhesion. These scaffolds were also immersed in an isopropanol bath to achieve complete crosslinking. Saraiva et al. [30] also used methacrylated-chitosan in combination to GelMA to obtain more stable structures and enhance mechanical properties derived from photopolymerization processes of both polymers. The printed scaffolds were biocompatible and supported surface cells adhesion and internalization.

Following other strategies, a different photosensitive chitosan was implemented by Bardakova et al. [31]. In this study, a chitosan-g-oligo (L,L-lactide) copolymer was synthesized and photopolymerized using two-photon polymerization induced by microstereolithography method. The authors envisioned that their $3 \mathrm{D}$ printed structures would be excellent candidates for treating spinal cord injuries by the straightforward application of light and a chitosan-based ink.

\section{D bioprinting of chitosan-based bioinks}

$3 \mathrm{D}$ bioprinting is an attractive biofabrication technique for the synthesis of intricate bioactive constructs since it allows the simultaneous deposition of living cells and bioactive compounds along with biomaterials $[10,32] .3 \mathrm{D}$ bioprinting is a very customizable technology through which cells can be deposited at precisely controlled locations to mimic native tissue architectures [32]. The combination of living cells and biomaterial solutions are termed bioinks and they must meet a number of requirements for their successful processability: (i) suitable rheological properties to guarantee filament deposition; (ii) adequate mechanical properties to ensure shape fidelity and avoid collapse between subsequent layers; 
(iii) biocompatibility to ensure cell viability during printing process; and (iv) excellent cytocompatibility to support cell proliferation and differentiation [3, 4, 29, 33]. The characteristic $\mathrm{pH}$ sensitivity of chitosan is the main disadvantage for its application in $3 \mathrm{D}$ bioprinting. The necessary acidic conditions to obtain an adequate solubility of the polymer are not cell friendly, therefore impeding cell encapsulation in the ink. For this reason, chitosan derivatives that are soluble at physiological conditions have been extensively developed and evaluated in the last years [16].

Carboxymethyl-chitosan (CMC) is an attractive chitosan derivative which exhibits excellent solubility properties at a $\mathrm{pH}$ range between 7-9. Thus, it has been widely applied in $3 \mathrm{D}$ bioprinting as it allows cell encapsulation. Huang et al. [32] used a polysaccharide bioink blend composed of $\mathrm{CMC}$, alginate, and gelatin together with bone mesenchymal stem cells (MSCs). The as-obtained 3D printed scaffolds were ionically crosslinked with $\mathrm{CaCl}_{2}$ solution. Moreover, the $3 \mathrm{D}$ constructs exhibited high cell viability (around 85\%) after 0 and 2 days of printing. In other work, $\mathrm{Gu}$ et al. [34] also used a similar polysaccharide combination for the 3D bioprinting of constructs that supported survival, proliferation and differentiation of human induced pluripotent stem cells (iPSCs). They extruded a bioink compounded by alginate, agarose, and CMC including iPSCs to fabricate 3D constructs that were crosslinked with $\mathrm{CaCl}_{2}$ solution. The authors claimed that the addition of CMC provided scaffolds with beneficial properties such as adequate porosity and permeability, low inflammatory reaction, antimicrobial responses, and it even enhanced biological features regarding cell differentiation.

$\beta$-glycerolphosphate ( $\beta$-GP) has opened the door for the application of novel thermosensitive chitosan-based hydrogel with spontaneous gelation at physiological temperature in different biofabrication techniques $[29,35]$. Although the crosslinking mechanism between chitosan and $\beta$-GP re- mains not well stablished, it is known that the addition of $\beta-G P$ to chitosan solution rises the $\mathrm{pH}$ to neutral value and the as-obtained hybrid hydrogel exhibits thermosensitiveness, being able to spontaneously form hydrogels at $37^{\circ} \mathrm{C}[17,36]$. Demirtas et al. [17] presented a bioprintable form of chitosan using $\beta$-GP for the first time. They successfully printed MC3T3-E1 preosteoblast cell-laden hydrogels by thermal crosslinking. Moreover, they claimed the superiority of chitosan respect to alginate regarding cell performance. The benefits of $\beta-\mathrm{GP} /$ chitosan combination were also taken by Roehm et al. [36], who optimized a 3D bioprinting approach using a chitosan-gelatin blend ink that included IMR-32 cells from neuroblastoma. Addition of $\beta-G P$ to the bioink allowed hydrogel formation at body temperature without the necessity of postprinting processes. In addition, 3D bioprinted scaffolds showed homogeneous cell distribution and high cell viability.

HBC is a water soluble and thermosensitive chitosan derivative that has been also applied in 3D bioprinting. HBC exhibits improved solubility properties at physiological conditions while maintaining chitosan biocompatibility properties, which eases cell encapsulation [37]. Li et al. [37] used HBC in combination with oxidized chondroitin sulfate hydrogel for cartilage tissue regeneration. In this work, blend bioink was crosslinked by Schiff-based reaction, and pluronic F127 acted as sacrificial mold to improve shape fidelity and resolution of the bioprinted structures. Encapsulated-human adipose-derived MSCs showed low inflammatory expression profile in vitro and weak inflammatory response in vivo.

Finally, a novel chitosan-cathecol bioink was recently developed by Lee et al. [38]. This bioink was directly printed in culture media, and vanadyl ions were added to improved mechanical properties of the constructs. The bioprinted systems having L929 encapsulated-fibroblasts showed high cell viability $(\approx$ $90 \%)$, demonstrating that this is a promising and step-forward 3D bioprinting methodology to fabricate stable constructs (Figure 3 ). 
a)

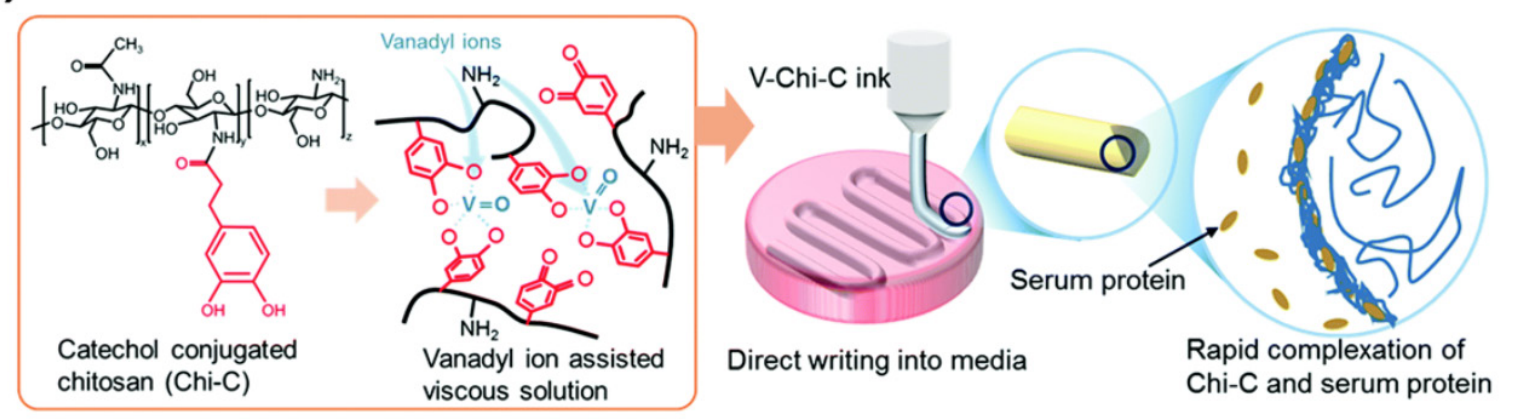

b)

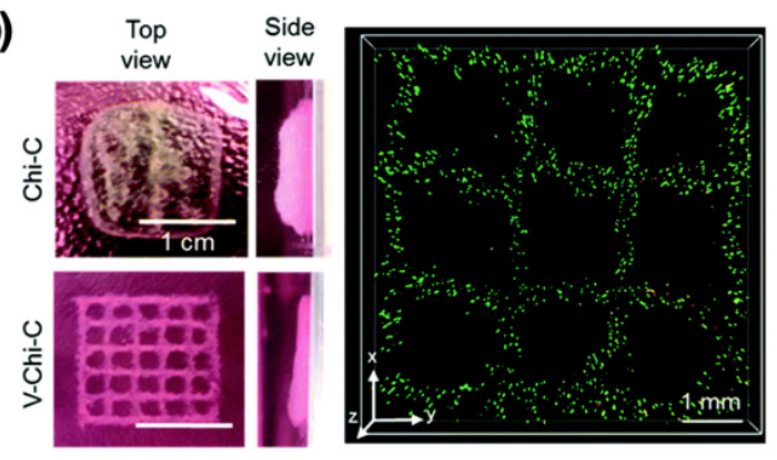

Figure 3. a) Schematic preparation of chitosan-catechol bioink and 3D bioprinting process; b) 3D bioprinted scaffolds pictures and live/dead assay performed on the scaffolds after printing process. Adapted from ref. [38] with permission from The Royal Society of Chemistry.

\section{Conclusions and future perspectives}

Undoubtedly, 3D printing and bioprinting have emerged as revolutionary technologies, promising to counteract conventional fabrication limitations. Current 3D printing mechanisms allow precise design and fabrication of intricate constructs through layerby-layer deposition of biomaterials and cells. However, the lack of printable hydrogel-based inks is hampering the progress of this technology for actual clinical applications. Chitosan has seized only a small area of its potential use in 3D printing and bioprinting fields due to its low printability properties. We present in this paper different chemical modifications of this polysaccharide or its combination with other compounds, which are attractive strategies to broaden its application. Currently, many efforts are being made on the development of new gelation mechanisms to improve not only chitosan processability and functionality, but also cell-encapsulation capacity. The summary of works presented in this paper can be a representative example of the substantial contribution chitosan can make to $3 \mathrm{D}$ printing field in terms of tissue regeneration, as a myriad of studies support its beneficial properties and key role in the repair of several damage tissues $[8,39]$. In conclusion, due to the transformation of 3D printing methodology to a more accessible technology, together with the tendency of using natural-derived materials, it can be predicted that chitosan-based inks will experience an increasing use in the next years.

\section{Acknowledgments}

Authors thank financial support to Ministry of Science, Innovation and Universities (Spain) (MAT2017-2017-84277-R), "La Caixa" Foundation (ID 100010434, scholarship of Ana Mora-Boza, code LCF/BQ/ ES16/11570018) and DAAD Research GrantsShort-term grants 2017. 


\section{References}

1. S. Derakhshanfar, R. Mbeleck, K. Xu, X. Zhang, W. Zhong, M. Xing, 3D bioprinting for biomedical devices and tissue engineering: $\mathrm{A}$ review of recent trends and advances, Bioact Mater 3(2) (2018) 144-156.

2. A.V. Do, B. Khorsand, S.M. Geary, A.K. Salem, 3D Printing of Scaffolds for Tissue Regeneration Applications, Adv Healthc Mater 4(12) (2015) 1742-62.

3. J. Gopinathan, I. Noh, Recent trends in bioinks for 3D printing, Biomater Res 22 (2018) 11.

4. J. Malda, J. Visser, F.P. Melchels, T. Jungst, W.E. Hennink, W.J. Dhert, J. Groll, D.W. Hutmacher, 25th anniversary article: Engineering hydrogels for biofabrication, Adv Mater 25(36) (2013) 501128.

5. X. Wang, C. Wei, B. Cao, L. Jiang, Y. Hou, J. Chang, Fabrication of Multiple-Layered Hydrogel Scaffolds with Elaborate Structure and Good Mechanical Properties via 3D Printing and Ionic Reinforcement, ACS Appl Mater Interfaces 10(21) (2018) 18338-18350.

6. Q. Wu, D. Therriault, M.-C. Heuzey, Processing and Properties of Chitosan Inks for 3D Printing of Hydrogel Microstructures, ACS Biomaterials Science \& Engineering 4(7) (2018) 2643-2652.

7. A. Mora-Boza, M.K. Włodarczyk-Biegun, A. del Campo, B. Vázquez-Lasa, J.S. Román, Glycerylphytate as an ionic crosslinker for 3D printing of multi-layered scaffolds with improved shape fidelity and biological features, Biomaterials Science 8(1) (2020) 506-516.

8. A. Muxika, A. Etxabide, J. Uranga, P. Guerrero, K. de la Caba, Chitosan as a bioactive polymer: Processing, properties and applications, Int J Biol Macromol 105(Pt 2) (2017) 1358-1368.

9. C.S.D. Cabral, S.P. Miguel, D. de Melo-Diogo, R.O. Louro, I.J. Correia, Green reduced graphene oxide functionalized $3 \mathrm{D}$ printed scaffolds for bone tissue regeneration, Carbon 146 (2019) 513523.

10. X. Hu, Y. Man, W. Li, L. Li, J. Xu, R. Parungao, Y. Wang, S. Zheng, Y. Nie, T. Liu, K. Song, 3D BioPrinting of CS/Gel/HA/Gr Hybrid Osteochondral Scaffolds, Polymers (Basel) 11(10) (2019).

11. S. Sayyar, S. Gambhir, J. Chung, D.L. Officer, G.G. Wallace, 3D printable conducting hydrogels containing chemically converted graphene, Nanoscale 9(5) (2017) 2038-2050.

12. P. Chavanne, S. Stevanovic, A. Wuthrich, $O$. Braissant, U. Pieles, P. Gruner, R. Schumacher, 3D printed chitosan / hydroxyapatite scaffolds for potential use in regenerative medicine, Biomed Tech (Berl) 58 Suppl 1 (2013).

13. S. Chen, Y. Shi, X. Zhang, J. Ma, Biomimetic synthesis of Mg-substituted hydroxyapatite nanocomposites and three-dimensional printing of composite scaffolds for bone regeneration, J Biomed Mater Res A 107(11) (2019) 2512-2521.

14. N.M. Ergul, S. Unal, I. Kartal, C. Kalkandelen, N. Ekren, O. Kilic, L. Chi-Chang, O. Gunduz, 3D printing of chitosan/ poly(vinyl alcohol) hydrogel containing synthesized hydroxyapatite scaffolds for hard-tissue engineering, Polymer Testing 79 (2019).

15. C. Bergonzi, A. Di Natale, F. Zimetti, C. Marchi, A. Bianchera, F. Bernini, M. Silvestri, R. Bettini, L. Elviri, Study of 3D-printed chitosan scaffold features after different post-printing gelation processes, Sci Rep 9(1) (2019) 362.

16. T.J. Kean, M. Thanou, Utility of Chitosan for 3D Printing and Bioprinting, Sustainable Agriculture Reviews 352019, pp. 271-292.

17. T.T. Demirtas, G. Irmak, M. Gumusderelioglu, A bioprintable form of chitosan hydrogel for bone tissue engineering, Biofabrication 9(3) (2017) 035003.

18. J. Zhang, B.J. Allardyce, R. Rajkhowa, Y. Zhao, R.J. Dilley, S.L. Redmond, X. Wang, X. Liu, 3D Printing of Silk Particle-Reinforced Chitosan Hydrogel Structures and Their Properties, ACS Biomaterials Science \& Engineering 4(8) (2018) 3036-3046.

19. J. Liu, L. Sun, W. Xu, Q. Wang, S. Yu, J. Sun, Current advances and future perspectives of $3 \mathrm{D}$ printing natural-derived biopolymers, Carbohydr Polym 207 (2019) 297-316.

20. C.R. Almeida, T. Serra, M.I. Oliveira, J.A. Planell, M.A. Barbosa, M. Navarro, Impact of 3-D printed PLA- and chitosan-based scaffolds on human monocyte/macrophage responses: unraveling the effect of 3-D structures on inflammation, Acta Biomater 10(2) (2014) 613-22.

21. L. Elviri, R. Foresti, C. Bergonzi, F. Zimetti, C. Marchi, A. Bianchera, F. Bernini, M. Silvestri, R. Bettini, Highly defined 3D printed chitosan scaffolds featuring improved cell growth, Biomed Mater 12(4) (2017) 045009.

22. C. Intini, L. Elviri, J. Cabral, S. Mros, C. Bergonzi, A. Bianchera, L. Flammini, P. Govoni, E. Barocelli, R. Bettini, M. McConnell, 3D-printed chitosan-based scaffolds: An in vitro study of human skin cell growth and an in-vivo wound healing evaluation in experimental diabetes in rats, Carbohydr Polym 199 (2018) 593-602.

23. F. Hafezi, N. Scoutaris, D. Douroumis, J. Boateng, 3D printed chitosan dressing crosslinked with genipin for potential healing of chronic wounds, Int J Pharm 560 (2019) 406-415.

24. I.H. Liu, S.H. Chang, H.Y. Lin, Chitosan-based hydrogel tissue scaffolds made by $3 \mathrm{D}$ plotting promotes osteoblast proliferation and mineralization, Biomed Mater 10(3) (2015) 035004.

25. A. Mora-Boza, M.L. López-Donaire, L. Saldaña, N. Vilaboa, B. Vázquez-Lasa, J. San Román, Glycerylphytate compounds with tunable ion affi- 
nity and osteogenic properties, Scientific Reports 9(1) (2019) 11491.

26. Q. Liu, Q. Li, S. Xu, Q. Zheng, X. Cao, Preparation and Properties of 3D Printed Alginate(-) Chitosan Polyion Complex Hydrogels for Tissue Engineering, Polymers (Basel) 10(6) (2018).

27. W.L. Ng, W.Y. Yeong, M.W. Naing, Polyelectrolyte gelatin-chitosan hydrogel optimized for $3 \mathrm{D}$ bioprinting in skin tissue engineering, International Journal of Bioprinting 2(0) (2016).

28. W.L. Ng, W.Y. Yeong, M.W. Naing, Development of Polyelectrolyte Chitosan-gelatin Hydrogels for Skin Bioprinting, Procedia CIRP 49 (2016) 105112.

29. D.F. Duarte Campos, A. Blaeser, A. Korsten, S. Neuss, J. Jakel, M. Vogt, H. Fischer, The stiffness and structure of three-dimensional printed hydrogels direct the differentiation of mesenchymal stromal cells toward adipogenic and osteogenic lineages, Tissue Eng Part A 21(3-4) (2015) 740-56.

30. S.M. Saraiva, S.P. Miguel, M.P. Ribeiro, P. Coutinho, I.J. Correia, Synthesis and characterization of a photocrosslinkable chitosan-gelatin hydrogel aimed for tissue regeneration, RSC Advances 5(78) (2015) 63478-63488.

31. K.N. Bardakova, T.S. Demina, E.A. Grebenik, N.V. Minaev, T.A. Akopova, V.N. Bagratashvili, P.S. Timashev, 3D printing biodegradable scaffolds with chitosan materials for tissue engineering, IOP Conference Series: Materials Science and Engineering 347 (2018).

32. J. Huang, H. Fu, Z. Wang, Q. Meng, S. Liu, H. Wang, X. Zheng, J. Dai, Z. Zhang, BMSCs-laden gelatin/sodium alginate/carboxymethyl chitosan hydrogel for 3D bioprinting, RSC Advances 6(110) (2016) 108423-108430.

33. M.K. Wlodarczyk-Biegun, A. Del Campo, 3D bioprinting of structural proteins, Biomaterials 134 (2017) 180-201.

34. Q. Gu, E. Tomaskovic-Crook, G.G. Wallace, J.M. Crook, 3D Bioprinting Human Induced Pluripotent Stem Cell Constructs for In Situ Cell Proliferation and Successive Multilineage Differentiation, Adv Healthc Mater 6(17) (2017).

35. S. Saravanan, S. Vimalraj, P. Thanikaivelan, S. Banudevi, G. Manivasagam, A review on injectable chitosan/beta glycerophosphate hydrogels for bone tissue regeneration, International Journal of Biological Macromolecules 121 (2019) 38-54.

36. K.D. Roehm, S.V. Madihally, Bioprinted chitosan-gelatin thermosensitive hydrogels using an inexpensive 3D printer, Biofabrication 10(1) (2017) 015002.

37. C. Li, K. Wang, X. Zhou, T. Li, Y. Xu, L. Qiang, M. Peng, Y. Xu, L. Xie, C. He, B. Wang, J. Wang, Controllable fabrication of hydroxybutyl chitosan/oxidized chondroitin sulfate hydrogels by $3 \mathrm{D}$ bioprinting technique for cartilage tissue engineering, Biomed Mater 14(2) (2019) 025006.

38. D. Lee, J.P. Park, M.Y. Koh, P. Kim, J. Lee, M. Shin, H. Lee, Chitosan-catechol: a writable bioink under serum culture media, Biomater Sci 6(5) (2018) 1040-1047.

39. F. Croisier, C. Jérôme, Chitosan-based biomaterials for tissue engineering, European Polymer Journal 49(4) (2013) 780-792. 